\title{
Scale Space Based Object-Oriented Shadow Detection and Removal from Urban High-Resolution Remote Sensing Images
}

\author{
Prof. Sagar Kothawade \\ Department of Electronics and Telecommunication Engineering, AGCOE India \\ sagarkothawade99@gmail.com
}

\begin{tabular}{|c|c|}
\hline Article History & Abstract \\
\hline $\begin{array}{l}\text { Article Submission } \\
18 \text { July } 2020 \\
\text { Revised Submission } \\
22 \text { October } 2020 \\
\text { Article Accepted } \\
21 \text { November } 2020 \\
\text { Article Published } \\
31^{\text {st }} \text { December } 2020\end{array}$ & $\begin{array}{l}\text { This task mostly center to get the high resolution color remote sensing image, and } \\
\text { furthermore attempted to eliminate the concealed district in the both metropolitan } \\
\text { and country region. A portion of the current activities are included to recognize the } \\
\text { concealed district and afterward dispense with that area, yet it has a few } \\
\text { disadvantages. The discovery of the edges will be influenced generally by the } \\
\text { utilization of the outside boundaries. The edge location cycle can be more useful in } \\
\text { the recognition of the articles with the goal that the items can be utilized for } \\
\text { additional handling. In this cycle we have execute the Scale Space algorithm is } \\
\text { utilized to identify the shadow area and concentrate the component from the shadow } \\
\text { district. Scale Space is least complex in area base image segmentation strategies. } \\
\text { The idea of Scale Space algorithm is check the neighboring pixels of the underlying } \\
\text { seed focuses. At that point decide if those neighboring pixels are added to the seed } \\
\text { focuses or not. In the Scale Space limit algorithm Pixels are set in the area } \\
\text { dependent on their properties or the properties of the close by pixel esteems. At that } \\
\text { point the pixel containing the comparable properties is gathered and afterward the } \\
\text { enormous quantities of pixels are circulated all through the image. } \\
\text { Keywords: Space Scale Algorithm, Shadow Detection }\end{array}$ \\
\hline
\end{tabular}

\section{Introduction}

Presently a day the man endure the huge region of the world, so to screen the land territory satellite imaging is utilized to recognize the earth region. The shadow is happened by the interfacing of building and the sun. In this cycle a portion of the issues happened, because of the shadow zone of a metropolitan and country territory. So this task fundamentally centre to get the high resolution color remote sensing image, and furthermore embraced to eliminate the concealed locale in the both metropolitan and provincial territory. Due to the surrounding light, the proportions of the two pixels are not same in each of the three color channels. These two pixels will be distinctive in force, yet additionally in tint and immersion. Along these lines, adjusting only the force of the shadowed pixels doesn't eliminate the shadow, and we need to address the chromaticity esteems too. Utilizing the shadow thickness, the shadow territory is sectioned into daylight, obscuration and umbra areas. Since the lighting color of the umbra locale isn't generally equivalent to that of the daylight area, the color change is performed between them. At that point, the color normal and difference of the umbra locale are changed in accordance with be equivalent to those of the daylight district. In the obscuration, color and brilliance changes for little Deb et al.: Shadow Detection and Removal Based on districts are played out equivalent to them are for the umbra locale. At long last, all limits between shadowed districts and neighbouring lit areas are smoothed by convolving them with a Gaussian veil.

\section{Space Scale Methods}

The principal district developing strategy was the cultivated Scale Space technique. This strategy accepts a bunch of seeds as contribution alongside the image. The seeds mark every one of the items to be fragmented. The districts are iteratively developed by contrasting all unallocated neighboring pixels with the locales. The 
distinction between a pixel's power esteem and the district's mean, is utilized as a proportion of comparability. The pixel with the littlest contrast estimated this way is allotted to the individual district. This cycle proceeds until all pixels are designated to a locale.

Cultivated Scale Space requires seeds as extra information. The segmentation results are reliant on the selection of seeds. Clamor in the image can make the seeds be ineffectively positioned. Unseeded Scale Space is a changed algorithm that doesn't need express seeds. It begins with a solitary district - the pixel picked here doesn't essentially impact last segmentation. At every emphasis it thinks about the neighboring pixels similarly as cultivated Scale Space. It contrasts from cultivated Scale Space in that in the event that the base is not exactly a predefined edge, at that point it is added to the individual area. On the off chance that not, at that point the pixel is considered altogether not quite the same as every single current locale and another area is made with this pixel.

One variation of this method, proposed by Haralick and Shapiro (1985), depends on pixel powers. The mean and disperse of the area and the force of the up-and-comer pixel is utilized to process a test measurement. In the event that the test measurement is adequately little, the pixel is added to the district, and the locale's mean and disperse are recomputed. Something else, the pixel is dismissed, and is utilized to shape another locale.

An exceptional locale developing technique is called - associated segmentation. It depends on pixel forces and neighbourhood-connecting ways. A level of network (connectedness) will be determined dependent on a way that is framed by pixels. For a specific estimation of, two pixels are called - associated if there is a way connecting those two pixels and the connectedness of this way is at any rate connectedness is an equality connection

\section{Proposed Methodology}

The methodology consist of four steps including pre-processing, feature extraction, segmentation and IOOPL matching.

In this pre-processing, the info image is under going to change over the RGB image into the dark scale image. The changed over dim scale image is resized into the particular resolution, subsequently the resolution is kept up till the further cycle. On the off chance that the commotion is happened in the image, at that point the exactness of the image is influence, so the channel is use to kill the clamour present in the image. Then the commotion free image is utilized for the further cycle.

In this strategy, the Pre-handling is done to resizing the image. Then the image is changed over into the grayscale image. The grayscale image is then changed over into the clamour free image by utilizing the channel 'medfilt2'. This sifted image is then changed over into the twofold image. The twofold image is just having the pixel estimations of 0 and 1 . The item locale is consider as a 1 and the dull region is consider as a 0 .

Highlight extraction is the way toward separating the necessary information's from the area of interest. In this strategy, the shadow include is separated by recognized with the Scale Space limit technique. At that point the item properties, for example, phantom highlights and mathematical highlights are joined with a spatial relationship, in which the bogus shadows are identified and disposed of. In this procedure, the element extraction is conveyed to remove the highlights of the 5 significant classifications. The Average grayscale estimation of the image is removed from the image. The pinnacle estimation of the histogram which is acquired by the shadow top histogram is extricated. At that point the limit estimation of the image is extricated. Simultaneously, the recurrence of the image is additionally removed from the image. Finally the close by pixel esteems are removed from a similar image from which the above highlights are separated

Segmentation is the way toward isolating the necessary part from the bunch of undesirable foundation. On different words, the segmentation is the cycle of end of the foundation area. To section the necessary item, the shape factor and the color factor is considered to eliminate the shadow, yet the dull locale of the image ought not be dispense with. The boundary of the each image is recorded and afterward the variety in the shadow and the dim territory is noted. 
IOOPL coordinating is a cycle of acquiring homogeneous areas by leading closeness coordinating to the IOOPL segment by segment. On the off chance that the connection coefficient is little, at that point there is some anomalous parts speaking to some various kinds of articles exist in this segment.

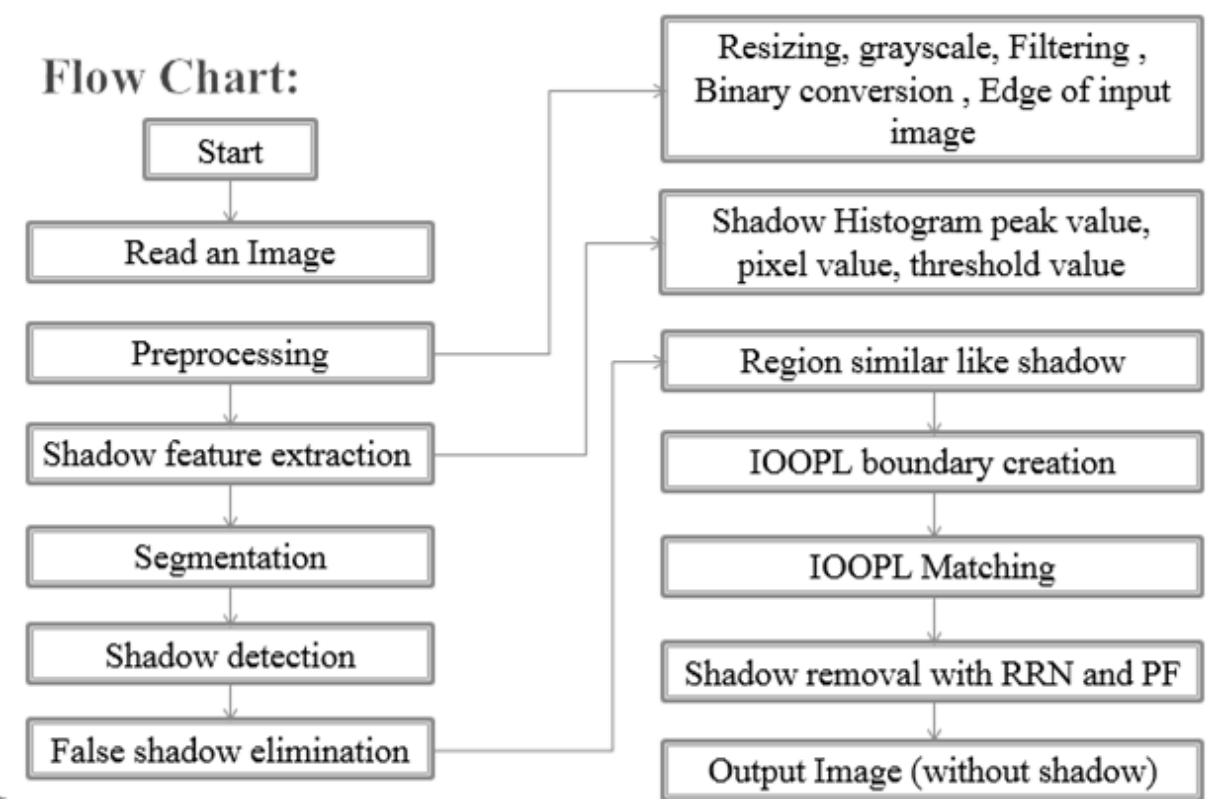

Fig 1. System Methodology Steps

In the Scale Space threshold algorithm Pixels are placed in the region based on their properties or the properties of the nearby pixel values. Then the pixel containing the similar properties is grouped together and then the large number of pixels is distributed throughout the image. We are proposed the future enhancement based on the Scale Space segmentation method. The aim of region detection is to provide the possibility to characterize the detected object by parameter analysis (shape, position, size...). Edge-based segmentation: borders between regions. Region-based segmentation: direct construction of regions. It is an easy way to construct regions from their borders and it is also easy to detect borders of existing regions. Segmentations resulting from edge-based methods and Scale Space methods are not usually exactly the same. Scale Space is simplest in region-base image segmentation methods. The concept of Scale Space algorithm is check the neighboring pixels of the initial seed points, then determine whether those neighboring pixels are added to the seed points or not.

\section{System Implementation and Results}

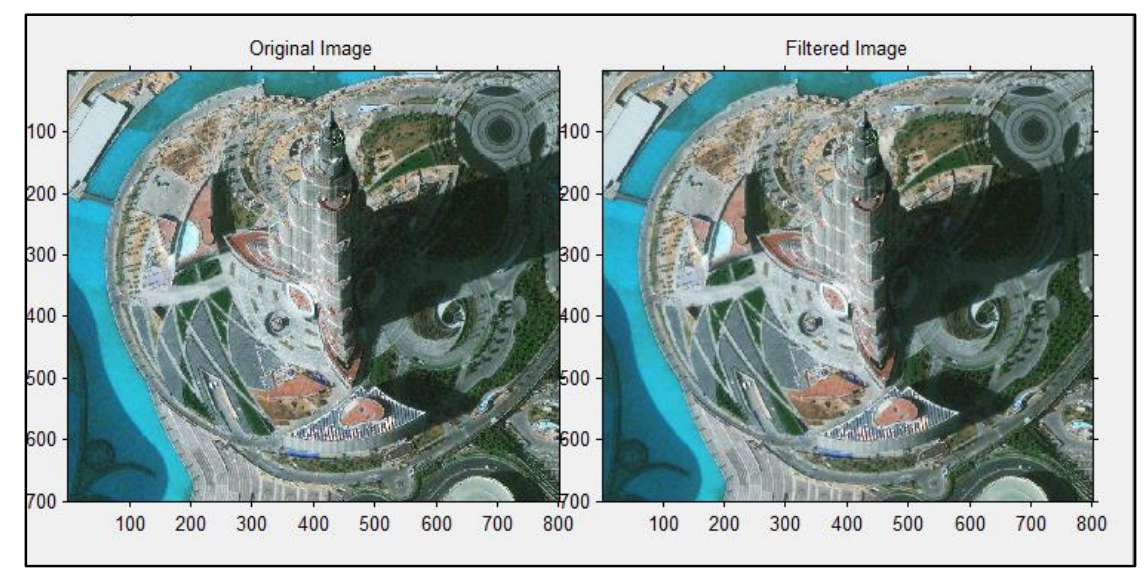

Fig 2. Filtering Input Image 


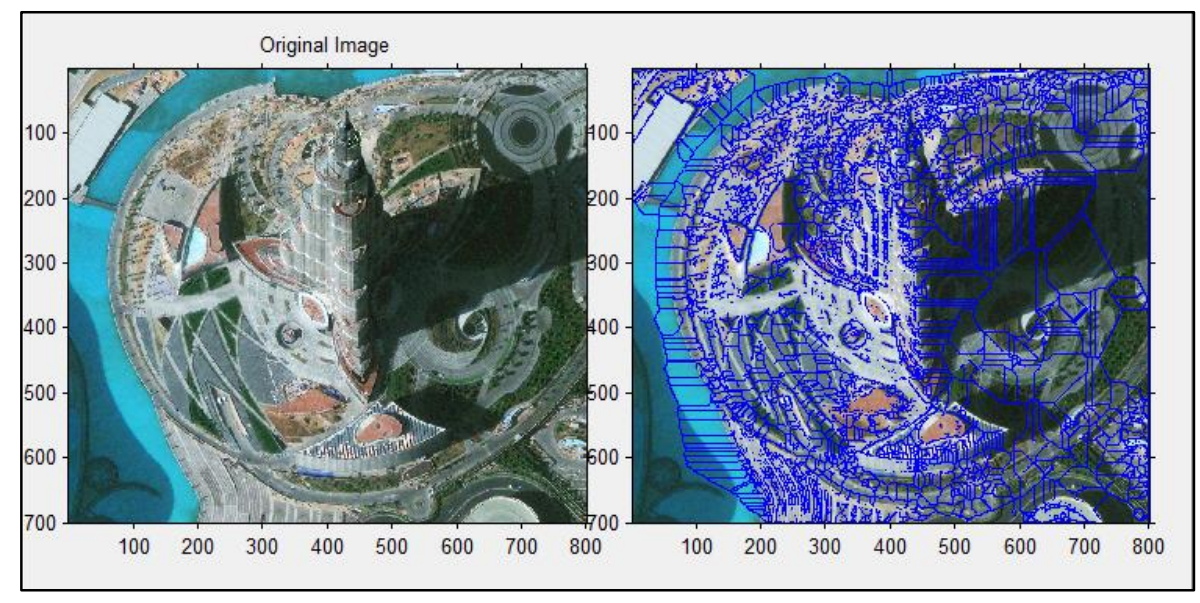

Fig 3. RGB Segmentation

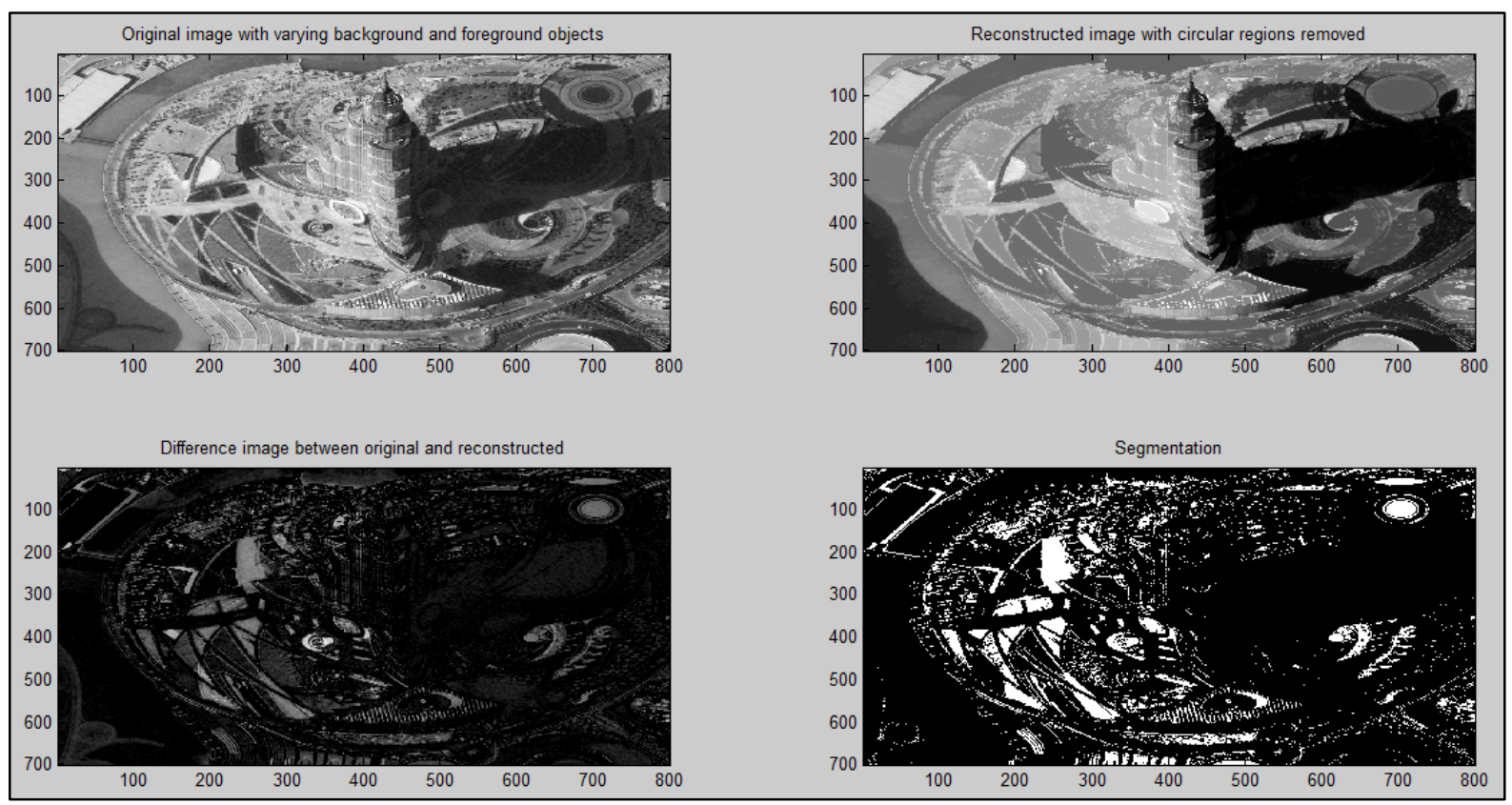

Fig 4. Segmentation of the image by the convexity along with the morphological

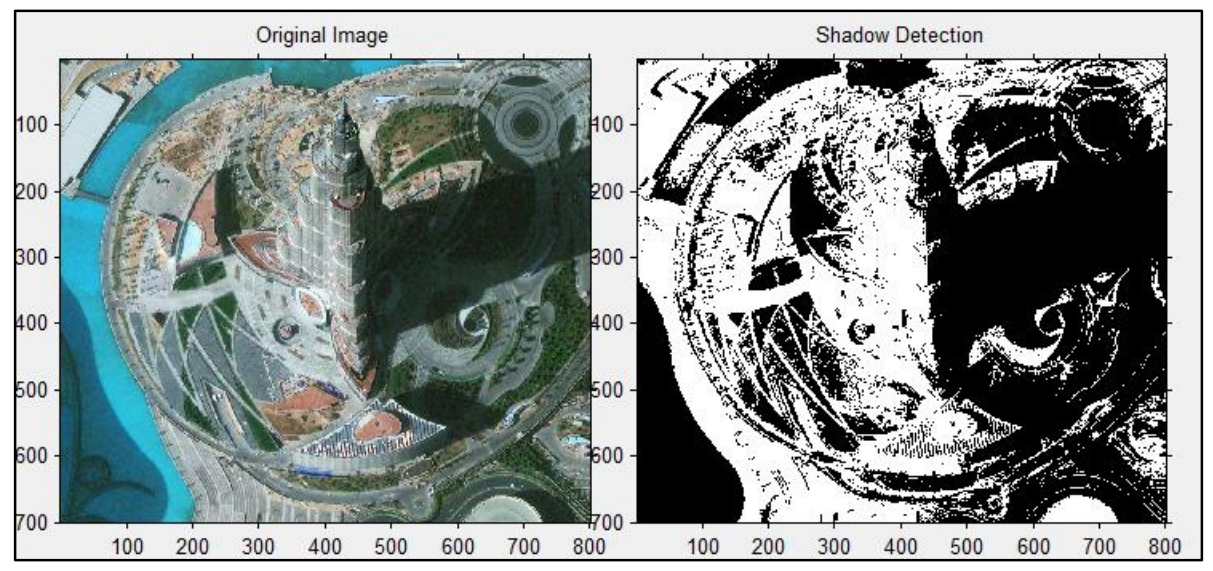

Fig 5. Shadow Detection using Thresholding 


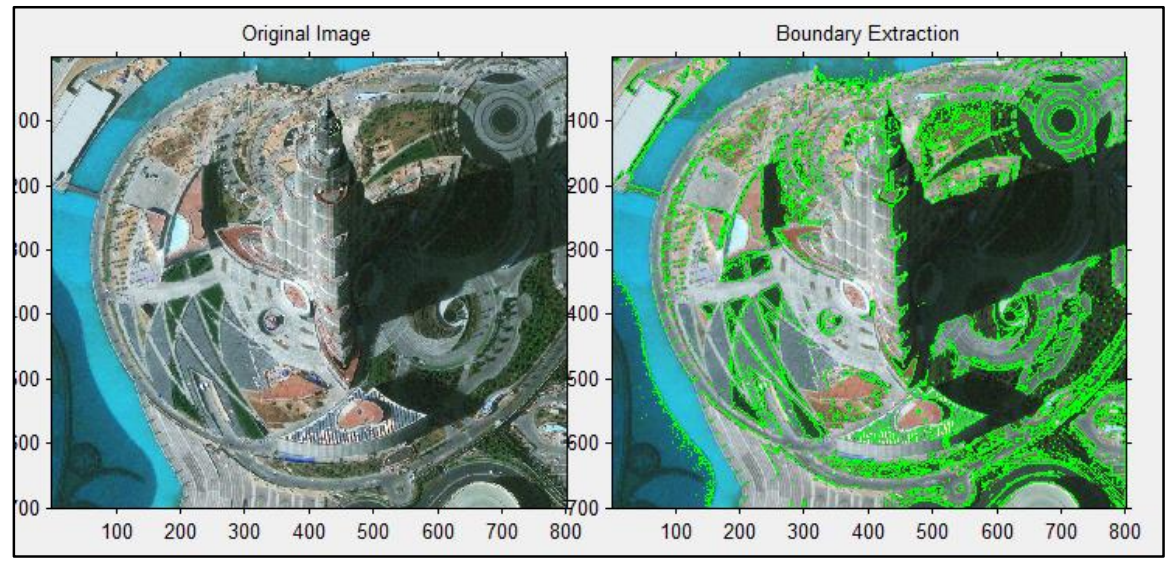

Fig 6. Boundary Extraction

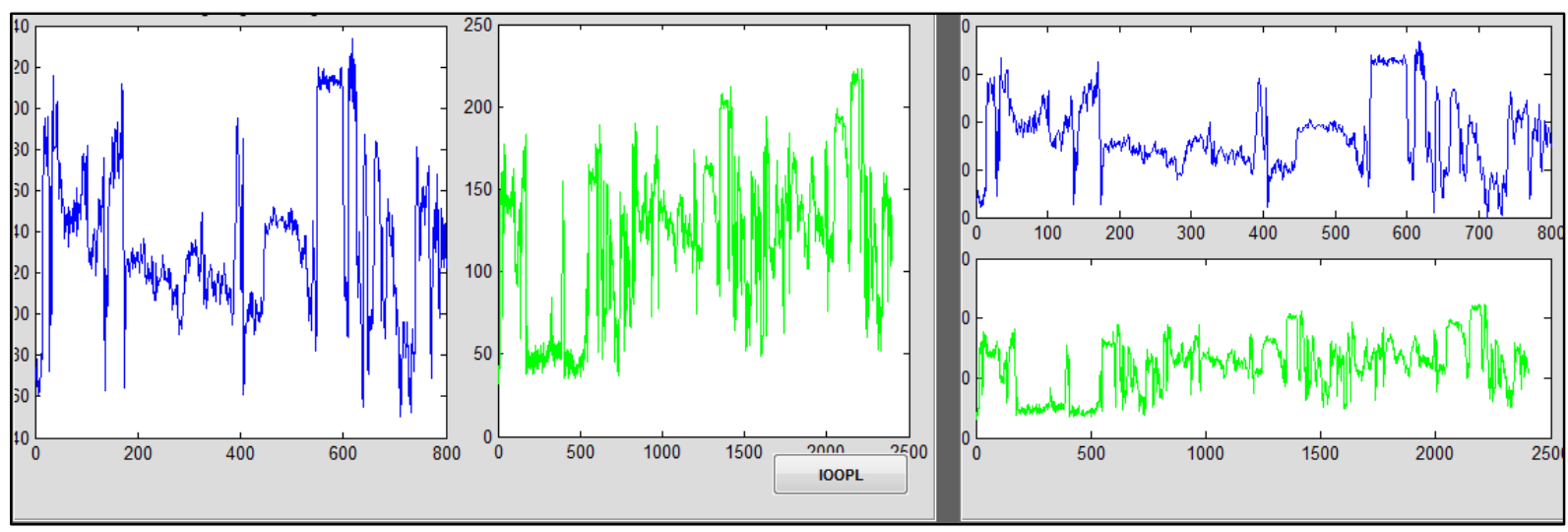

Fig 7. Graphical Representation after IOOPL

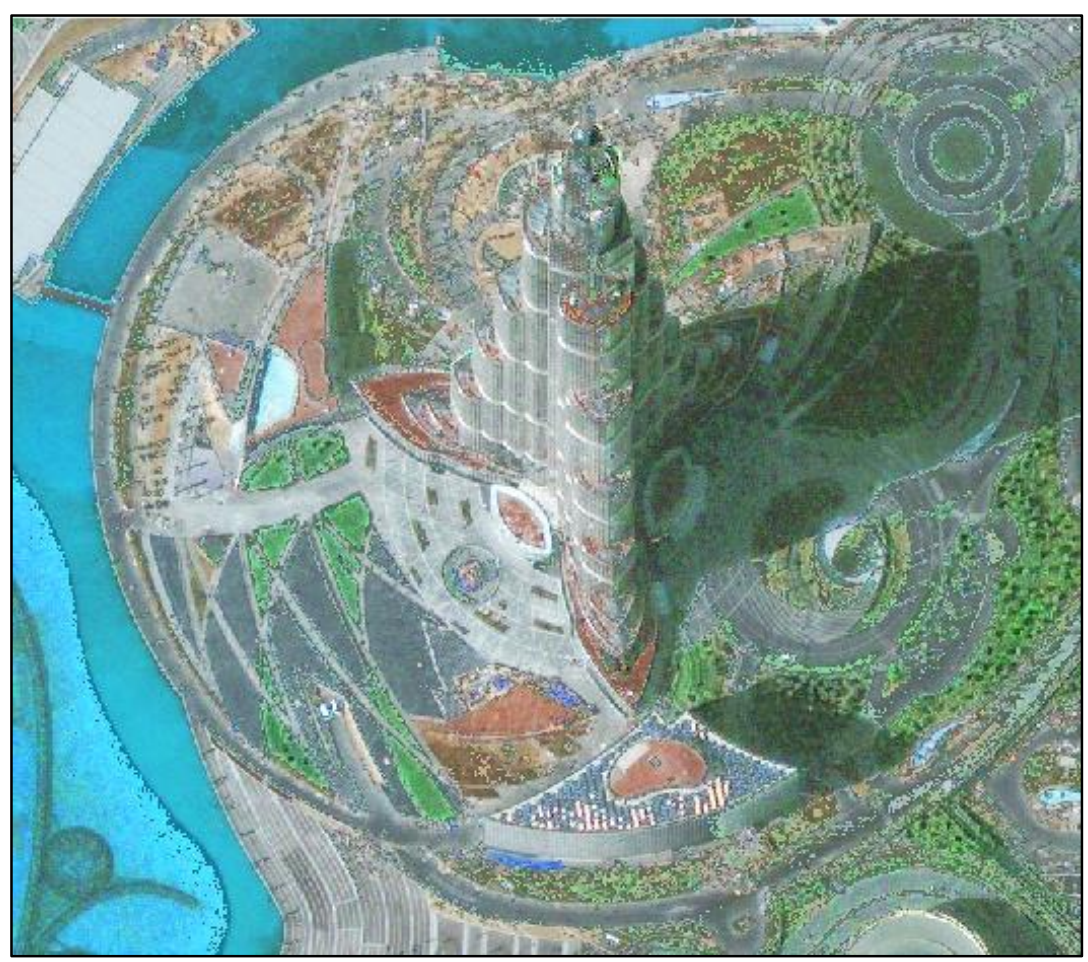

Fig 8. Final Shadow Extracted Image 


\section{Conclusion}

Hence the shadow highlight is extricated from the image and afterward the undesirable shadow got in the image is taken out from the image. The image nature of the image is acquired. In all the change recognition applications dependent on remote sensing images, various reasons for application centre on various change data, so there is no general change identification technique. Focused on such trademark, this paper has summed up the change types during change location and has dealt with the item includes, in view of which a multi-highlight coordinated article level change recognition strategy considering motivations behind applications and properties of various kinds of item has been advanced

\section{References}

[1] T. Kim, T. Javzandulam, and T.-Y. Lee, "Semiautomatic reconstruction of building height and footprints from single satellite images," in Proc. IGARSS, Jul. 2007, vol. 2, pp. 4737-4740.

[2] S. Ji and X. Yuan, "A method for shadow detection and change detection of man-made objects," J. Remote Sens., vol. 11, no. 3, pp. 323-329, 2007.

[3] P.M. Dare, "Shadow analysis in high-resolution satellite imagery of urban areas," Photogramm. Eng. Remote Sens., vol. 71, no. 2, pp. 169-177, 2005.

[4] Y. Li, P. Gong, and T. Sasagawa, "Integrated shadow removal based on photogrammetry and image analysis," Int. J. Remote Sens., vol. 26, no. 18, pp. 3911-3929, 2005.

[5] W. Zhou, G. Huang, A. Troy, and M. L. Cadenasso, "Object-based land cover classification of shaded areas in high spatial resolution imagery of urban areas: A comparison study,” Remote Sens. Env., vol. 113, no. 8, pp. 1769-1777, 2009.

[6] J. Yoon, C. Koch, and T. J. Ellis, "ShadowFlash: An approach for shadow removal in an active illumination environment," in Proc. 13th BMVC, Cardiff, U.K., Sep. 2-5, 2002, pp. 636-645.

[7] R. B. Irvin and D. M. McKeown, Jr, "Methods for exploiting the relationship between buildings and their shadows in aerial imagery," IEEE Trans. Syst., Man, Cybern., vol. 19, no. 6, pp. 1564-1575, Dec. 1989.

[8] Y. Li, T. Sasagawa, and P. Gong, "A system of the shadow detection and shadow removal for high resolution city aerial photo," in Proc. ISPRS Congr, Comm., 2004, vol. 35, pp. 802-807, Part B3.

[9] R. Highnam and M. Brady, "Model-based image enhancement of far infrared images," IEEE Trans Pattern Anal. Mach. Intell., vol. 19, no. 4, pp. 410-415, Apr. 1997.

[10] G. Finlayson, S. Hordley, and M. Drew, "Removing shadows from images," in Proc. ECCV, May 2831, 2002, pp. 823-836, Vision-Part IV.

[11] E. Salvador, A. Cavallaro, and T. Ebrahimi, "Shadow identification and classification using invariant color models," in Proc. IEEE Int. Conf. Acoust., Speech, Signal Process., 2001, vol. 3, pp. 1545-1548.

[12] V. J. D. Tsai, "A comparative study on shadow compensation of color aerial images in invariant color models," IEEE Trans. Geosci. Remote Sens., vol. 44, no. 6, pp. 1661-1671, Jun. 2006.

[13] K.-L. Chung, Y.-R. Lin, and Y.-H. Huang, "Efficient shadow detection of color aerial images based on successive scheme," IEEE Trans. Geosci. Remote Sens., vol. 47, no. 2, pp. 671-682, Feb. 2009.

[14] D. Cai, M. Li, Z. Bao et al., "Study on shadow detection method on high resolution remote sensing image based on HIS space transformation and NDVI index," in Proc. 18th Int. Conf. Geoinformat., Jun. 2010, pp. 1-4.

[15] H. Ma, Q. Qin, and X. Shen, "Shadow segmentation and compensation in high resolution satellite images," in Proc. IEEE IGARSS, Jul. 2008, vol. 2, pp. 1036-1039.

[16] J. Yang, Z. Zhao, and J. Yang, "A shadow removal method for high resolution remote sensing image," Geomatics Inf. Sci. Wuhan Univ., vol. 33, no. 1, pp. 17-20, 2008.

[17] Z. Zhu and C. E. Woodcock, "Object-based cloud and cloud shadow detection in Landsat imagery," Remote Sens. Environ., vol. 118, pp. 83-94, 2012.

[18] Makarau, R. Richter, R. Muller et al., "Adaptive shadow detection using a blackbody radiator model," IEEE Trans. Geosci. Remote Sens., vol. 49, no. 6, pp. 2049-2059, 2011. 
[19] L. Lorenzi, F. Melgani, and G. Mercier, "A complete processing chain for shadow detection and reconstruction in VHR images," IEEE Trans. Geosci. Remote Sens., vol. 50, no. 9, pp. 3440-3452, 2012.

[20] P. Sarabandi, F. Yamazaki, M. Matsuoka et al., "Shadow detection and radiometric restoration in satellite high resolution images," in Proc. IEEE IGARSS, Sep. 2004, vol. 6, pp. 3744-3747. 